Pacific Journal of Mathematic 


\section{COMPACT SUBSETS OF A TYCHONOFF SET}

\section{Geoffrey Fox and Pedro Morales}

The paper establishes a relation between the partial exponential law and the compactness of certain subsets of Tychonoff sets of multifunctions, and deduces consequences bearing on the Ascoli theorems established by Weston and Lin-Rose.

1. Introduction. The "Tychonoff set" is an abstraction of a class of sets arising in extensions of the classical Tychonoff theorem to multifunction context ([2], [5]). Extending the definition of the partial exponential law to multifunctions, we show that, when it is satisfied for a topology $\tau$, certain subsets of a Tychonoff set are $\tau$-compact. This approach - which is a non-trivial modification of the method introduced into function Ascoli theory by Noble [7] - will yield, in particular, sufficient conditions for compactness relative to the compact open topology.

In [6] Lin and Rose introduced a multifunction extension of the Kelley-Morse notion of even continuity, and proved a multifunction Ascoli theorem of the Weston type, without, however, showing that it contains the prototype [11, p. 20]. We deduce from our criterion a generalization of the Lin-Rose theorem. We show that this generalization contains the Weston Ascoli theorem and yields corollaries equivalent to the Tychonoff theorems for point-compact and point-closed multifunctions established in [2].

2. Multifunctions. We review the established definitions for multifunctions ([1], [9],[10]): Let $X, Y$ be nonempty sets. A multifunction is a point to set correspondence $f: X \rightarrow Y$ such that, for all $x \in X, f x$ is a nonempty subset of $Y$. For $A \subseteq X, B \subseteq Y$ it is customary to write $f(A)=\bigcup_{x \in A} f x, f^{-}(B)=\{x: x \in X$ and $f x \cap B \neq \phi\}$ and $f^{+}(B)=\{x: x \in X$ and $f x \subseteq B\}$. If $Y$ is a topological space, a multifunction $f: X \rightarrow Y$ is point-compact (point-closed) if $f x$ is compact (closed) for all $x \in X$. If $X, Y$ are topological spaces, a multifunction $f: X \rightarrow Y$ is continuous if $f^{-}(U), f^{+}(U)$ are open in $X$ whenever $U$ is open in $Y$. Henceforth the set of all continuous multifunctions (continuous functions) on a topological space $X$ to a topological space $Y$ will be denoted $\mathscr{C}(X, Y)(C(X, Y))$.

Let $\left\{Y_{x}\right\}_{x \in X}$ be a family of nonempty sets. The m-product $P\left\{Y_{x}: x \in X\right\}$ of the $Y_{x}$ is the set of all multifunctions $f: X \rightarrow \cup_{x \in X} Y_{x}$ 
such that $f x \subseteq Y_{x}$ for all $x \in X$. In the case $Y_{x}=Y$ for all $x \in X$, the $m$-product of the $Y_{x}$, denoted $Y^{m X}$, is the set of all multifunctions on $X$ to $Y$. For $x \in X$, the $x$-projection $p r_{x}: P\left\{Y_{x}: x \in X\right\} \rightarrow Y_{x}$ is the multifuntion defined by $p r_{x} f=f x$. If the $Y_{x}$ are topological spaces, the pointwise topology $\tau_{p}$ on $P\left\{Y_{x}: x \in X\right\}$ is defined to be the topology having as open subbase the sets of the forms $p r_{x}^{-}\left(U_{x}\right), p r_{x}^{+}\left(U_{x}\right)$, where $U_{x}$ is open in $Y_{x}, x \in X([5],[8])$.

For $F \subseteq Y^{m x}, x \in X$, we write $F[x]=\cup_{f \in F} f x$. Let $Y$ be a topological space. We say that a subset $F$ of $Y^{m X}$ is pointwise bounded if $F[x]$ has compact closure in $Y$ for all $x \in X$. We say that a subset $T$ of $Y^{m X}$ is Tychonoff if, for every pointwise bounded subset $F$ of $T$, $T \cap P\{\overline{F[x]}: x \in X\}$ is $\tau_{p}$-compact. The following subsets of $Y^{m X}$ are Tychonoff:

(1) $Y^{X}$, by the classical Tychonoff theorem.

(2) $Y^{m X}$, by the theorem of $\operatorname{Lin}[5$, p. 400].

(3) The set of all point-closed members of $Y^{m x}$, by Corollary 2 of [2].

(4) The set of all point-compact members of $Y^{m x}$, by Corollary 3 of [2].

LEMMA 2.1. If $F$ is a pointwise bounded subset of a Tychonoff set $T$, then the $\tau_{p}$-closure of $F$ is compact.

Proof. Let $\bar{F}$ denote the $\tau_{p}$-closure of $F$. Since $P\{\overline{F[x]}: x \in$ $X\} \cap T$ is a $\tau_{p}$-compact subset of $T$, if suffices to show that $\bar{F} \subseteq$ $P\{\overline{F[x]:} x \in X\}$. Let $f \in \bar{F}$. We must show that, for $x \in X, y \in f x$ and an open neighbourhood $V$ of $y, F[x] \cap V \neq \varnothing$. Since $M=$ $\{h: h \in T$ and $h x \cap V \neq \varnothing\}$ is a $\tau_{p}$-neighborhood of $f$, there exists $h^{\prime} \in M \cap F$. Then $h^{\prime} x \cap V \neq \varnothing$ and $h^{\prime} x \subseteq F[x]$, so $F[x] \cap V \neq \varnothing$.

Let $X, Y$ be topological spaces. The multifunction $(f, x) \rightarrow f x$ on $Y^{m X} \times X$ to $Y$, or any restriction, will be denoted by the symbol $\omega$. Let $F \subseteq Y^{m X}$. A topology $\tau$ on $F$ is said to be jointly continuous if $\omega:(F, \tau) \times X \rightarrow Y$ is continuous [8, p. 48]. The compact open topology $\tau_{c}$ on $Y^{m X}$ is defined to be the topology having as open subbase the sets of the forms $\{f: f(K) \subseteq U\},\{f: f x \cap U \neq \varnothing$ for all $x \in K\}$, where $K$ is a compact subset of $X$ and $U$ is open in $Y$ ([6, p. 742], [8, p. 47]). Obviously $\tau_{c}$ is larger than $\tau_{p}$.

3. Partial exponential law.Let $X, Y, Z$ be topological spaces. An element $f \in Z^{m(X \times Y)}$ determines the function $\tilde{f}: x \rightarrow f(x, \cdot)$ on $X$ to $Z^{m Y}$. The function $\mu: f \rightarrow \tilde{f}$, called the exponential map, is a bijection of $Z^{m(X \times Y)}$ onto $\left(Z^{m Y}\right)^{X}$. It is clear that if $f \in \mathscr{C}(X \times Y, Z)$, then $\tilde{f}(x)=f(x, \cdot) \in \mathscr{C}(Y, Z)$ for all $x \in X$. When $\tau$ is a topology on 
$Z^{m Y}$, we say that $(X, Y, Z, \tau)$ satisfies the partial exponential law if $\mu(\mathscr{C}(X \times Y, Z)) \subseteq C(X,(\mathscr{C}(Y, Z), \tau))$.

We establish now the main theorem of the paper:

THEOREM 3.1. Let $T$ be a Tychonoff set of multifunctions on a topological space $X$ to a topological space $Y$, and let $\tau$ be a topology on $Y^{m X}$ such that $(K, X, Y, \tau)$ satisfies the partial exponential law for all compact spaces $K$. Then a subset $F$ of $T$ is $\tau$-compact if

(a) $F$ is $\tau$-closed in $T$,

(b) $F$ is pointwise bounded, and

(c) $\tau_{p}$ is jointly continuous on the $\tau_{p}$-closure of $F$ in $T$.

Proof. Let $\bar{F}$ denote the $\tau_{p}$-closure of $F$ in $T$ and let $\omega:\left(\bar{F}, \tau_{p}\right) \times$ $X \rightarrow Y$. By (c), $\omega$ is continuous, so $\bar{F} \subseteq \mathscr{C}(X, Y)$. Since $T$ is a Tychonoff set, (b) implies, by Lemma 2.1 , that $\bar{F}$ is $\tau_{p}$-compact. Then $\tilde{\omega}:\left(\bar{F}, \tau_{p}\right) \rightarrow(\mathscr{C}(X, Y), \tau)$ is continuous. Since $\tilde{\omega}$ is the inclusion map, $\bar{F}=\tilde{\omega}(\bar{F})$ is $\tau$-compact. Since, by (a), $F$ is $\tau$-closed in $\bar{F}$, it follows that $F$ is $\tau$-compact.

The application of this theorem to $\tau_{c}$ depends on the following generalization to multifunctions of Lemma 1 of R. H. Fox [3, p. 430]:

Lemma 3.2. (X, Y, $\left.Z, \tau_{c}\right)$ satisfies the partial exponential law.

Proof. Let $f \in \mathscr{C}(X \times Y, Z)$. Let $x \in X$. Since $f(x, \cdot)=f \circ j$, where $j(y)=(x, y)(y \in Y), f(x, \cdot)$ is continuous [9, p. 35]. Thus $\tilde{f}$ maps $X$ into $\mathscr{C}(Y, Z)$. It remains to show that $\tilde{f}: X \rightarrow\left(\mathscr{C}(Y, Z), \tau_{c}\right)$ is continuous.

Let $M=\{h: h \in \mathscr{C}(Y, Z)$ and $h(K) \subseteq U\}$, where $K$ is a compact subset of $Y$ and $U$ is open in $Z$. Let $x_{0} \in \tilde{f}^{-1}(M)$. Then $f\left(x_{0}, \cdot\right) \in M$, so $\left\{x_{0}\right\} \times K \subseteq f^{+}(U)$. By the theorem of Wallace [4, p. 142], there is a neighbourhood $V$ of $x_{0}$ such that $V \times K \subseteq f^{+}(U)$. Let $x \in V$. Then, for all $y \in K, \tilde{f}(x) y=f(x, y) \subseteq U$, so $\tilde{f}(x)(K) \subseteq U$. Thus $x \in \tilde{f}^{-1}(M)$, and we have shown that $\tilde{f}^{-1}(M)$ is open in $X$.

Let $M=\{h: h \in \mathscr{C}(Y, Z)$ and $h y \cap U \neq \varnothing$ for all $y \in K\}$, where $K$ is a compact subset of $Y$ and $U$ is open in $Z$. Let $x_{0} \in \tilde{f}^{-1}(M)$. Then $f\left(x_{0}, \cdot\right) \in M$, so $\left\{x_{0}\right\} \times K \subseteq f^{-}(U)$. There is a neighbourhood $V$ of $x_{0}$ such that $V \times K \subseteq f^{-}(U)$. Let $x \in V$. Then, for all $y \in K, \tilde{f}(x) y \cap$ $U \neq \varnothing$, so $\tilde{f}(x) \in \bar{M}$, that is, $x \in \tilde{f}^{-1}(M)$, and we have shown that $\tilde{f}^{-1}(M)$ is open in $X$.

4. Even continuity. Let $X, Y$ be topological spaces and let $F \subseteq Y^{m X}$. Following [6], we say that $F$ is evenly continuous if, for each 
$(x, y) \in X \times Y$ and each neighborhood $V$ of $y$, there exist neighbourhoods $U, W$ of $x, y$, respectively, such that

(a) $f \in F$ and $f x \cap W \neq \varnothing$ imply $U \subseteq f^{-}(V)$, and

(b) $f \in F, f x \cap W \neq \varnothing$ and $f x \subseteq V$ imply $f(U) \subseteq V$.

Lemma 4.1. Let $X, Y$ be topological spaces and let $F \subseteq Y^{m x}$. If $F$ is evenly continuous, $\tau_{p}$ on $F$ is jointly continuous.

Proof. Let $\omega:\left(F, \tau_{P}\right) \times X \rightarrow Y$. Suppose that $(f, x) \in \omega^{-}(V)$, where $V$ is open in $Y$. Choose $y \in f x \cap V$. Then there exist open neighbourhoods $U, W$ of $x, y$, respectively, such that $g \in F$ and $g x \cap$ $W \neq \varnothing \quad$ imply $U \subseteq g^{-}(V)$. Write $\quad M=\{h: h \in F \quad$ and $h x \cap W \neq \varnothing\}$. Then $M \times U$ is a neighbourhood of $(f, x)$, which is contained in $\omega^{-}(V)$. Now suppose that $(f, x) \in \omega^{+}(V)$, where $V$ is open in $Y$. Then $f x \subseteq V$. Choose $y \in f x$. There exist open neighbourhoods $U, W$ of $x, y$, respectively, such that $g \in F, g x \cap W \neq \varnothing$ and $g x \subseteq V$ imply $g(U) \subseteq V$. Write $M=\{h: h \in F, h x \cap W \neq \varnothing$ and $h x \subseteq V\}$. Then $M \times U$ is a neighbourhood of $(f, x)$, which is contained in $\omega^{+}(V)$.

Corollary 4.2. Let $X, Y$ be topological spaces and let $F \subseteq$ $Y^{m X}$. If $F$ is evenly continuous, then each member of $F$ is continuous.

The following result, which generalizes the Ascoli theorem of Lin and Rose [6, p. 746], contains also the Weston Ascoli theorem [11, p. 20]:

THEOREM 4.3. Let $T$ be a Tychonoff set of multifunctions on a topological space $X$ to a topological space $Y$. Then a subset $F$ of $T$ is $\tau_{c}$-compact if

(a) $F$ is $\tau_{c}$-closed,

(b) $F$ is pointwise bounded, and

(c) $F$ is evenly continuous.

Proof. By Lemma 3.2, this theorem will follow as a corollary of Theorem 3.1 if we show that $\tau_{p}$ is jointly continuous on the $\tau_{p}$-closure $\bar{F}$ of $F$. By (c) and Lemma 4.1, $\tau_{p}$ on $F$ is jointly continuous. By (a), $F=\tilde{F}$, where $\tilde{F}$ is the $\tau_{c}$-closure of $F$. Finally, by (c) and Lemma 3.1 of [6, p. 744], $\tilde{F}=\bar{F}$.

Corollary 4.4. Let $\left(Y^{m X}\right)_{0}\left(\left(Y^{m x}\right)_{1}\right)$ be the set of all pointcompact (point-closed) multifunctions on a topotogical space $X$ to a 
topological space $Y$. Then a subset $F$ of $\left(Y^{m X}\right)_{0}\left(\left(Y^{m X}\right)_{1}\right)$ is $\tau_{c}$-compact if
(a) $F$ is $\tau_{c}$-closed,
(b) $F$ is pointwise bounded, and
(c) $F$ is evenly continuous.

5. Remarks. The Lin-Rose Ascoli theorem [6, p. 746], depends, apart from Lemma 3.1 of [6], on the Tychonoff theorem of Lin [5, p. 400]. Consequently, the Corollary 4.4 can be proved by the Lin-Rose argument, using the Tychonoff theorems of [2]. We will prove the converse implication: Let $\left\{Y_{x}\right\}_{x \in X}$ be a family of compact spaces. We will deduce from Corollary 4.4 that $F=\left(P\left\{Y_{x}: x \in X\right\}\right)_{0}$ is $\tau_{p}$ - compact.

We may suppose the $Y_{x}$ disjoint. Assign to $X$ the discrete topology and let $Y=\cup_{x \in X} Y_{x}$ have the sum topology. We have $F \subseteq\left(Y^{m X}\right)_{0}$ and, since $X$ is discrete, $F$ is evenly continuous $[6, \mathrm{p}$. 743]. Since $F[x]=Y_{x}$ and $Y_{x}$ is closed in $Y, F$ is pointwise bounded. If we show that $F$ is $\tau_{p}$-closed, it will follow from Corollary 4.4 that $F$ is $\tau_{c}$-compact and therefore $\tau_{p}$-compact. Let $\left\{f_{\alpha}\right\}$ be a net in $F$ which is $\tau_{p}$-convergent to an element $f \in\left(Y^{m x}\right)_{0}$. Let $x \in X$. Let $y \in f x$, and let $V$ be an open neighborhood of $y$. Since $\left\{h: h \in\left(Y^{m x}\right)_{0}\right.$ and $h x \cap V \neq \varnothing\}$ is a $\tau_{p}$-neighborhood of $f, f_{\alpha} x \cap V \neq \varnothing$ eventually. Since $f_{\alpha} x \subseteq Y_{x}, Y_{x} \cap V \neq \varnothing$. This shows that $y \in \bar{Y}_{x}=Y_{x}$, proving that $f \in F$.

We prove similarly the same implication for $F=\left(P\left\{Y_{x}: x \in X\right\}\right)_{1}$.

\section{REFERENCES}

1. C. Berge, Topological Spaces, The MacMillan Company, New York (1965).

2. G. Fox and P. Morales, A general Tychonoff theorem for multifunctions, to appear in Canad. Math. Bull.

3. R. H. Fox, On topologies for function spaces, Bull. Amer. Math. Soc., 51 (1945), 429-432.

4. J. Kelley, General Topology, D. Van Nostrand, New York (1965).

5. Y. F. Lin, Tychonoff's theorem for the space of multifunctions, Amer. Math. Monthly, 74 (1967), 399-400.

6. Y. F. Lin and D. A. Rose, Ascoli theorem for spaces of multifunctions, Pacific J. Math., 34 (1970), 741-747.

7. N. Noble, Ascoli theorems and the exponential map, Trans. Amer. Math. Soc., 143 (1969), 393-411.

8. R. E. Smithson, Topologies on sets of relations, J. Nat. Sci. and Math. (Lahore), 11 (1971), 43-50.

9. _ Multifunctions, Nieuw. Arch. Wisk., 20 (1972), 31-53.

10. W. L. Strother, Continuous multi-valued functions, Bol. Soc. Mat. São Paulo, 10 (1955), 87-120.

11. J. D. Weston, A generalization of Ascoli's theorem, Mathematika, 6 (1959), 19-24.

Received April 24, 1974.

UNIVERSITÉ DE MONTRÉAL 

D. E. Bennett, Strongly unicoherent continua ............................. 1

Walter R. Bloom, Sets of p-spectral synthesis ................................ 7

R. T. Bumby and D. E. Dobbs, Amitsur cohomology of quadratic extensions: Formulas and number-theoretic examples ................. 21

W. W. Comfort, Compactness-like properties for generalized weak topological sums

D. R. Dunninger and J. Locker, Monotone operators and nonlinear biharmonic boundary value problems ...

T. S. Erickson, W. S. Martindale, 3rd and J. M. Osborn, Prime nonassociative algebras

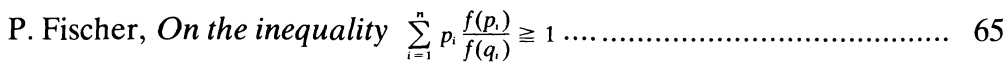

G. Fox and P. Morales, Compact subsets of a Tychonoff set ............... 75

R. Gilmer and J. F. Hoffmann, A characterization of Prüfer domains in terms of polynomials ......................................................... 81

L. C. Glaser, On tame Cantor sets in spheres having the same projection in each direction ......................................................... 87

Z. Goseki, On semigroups in which $X=X Y X=X Z X$ if and only if

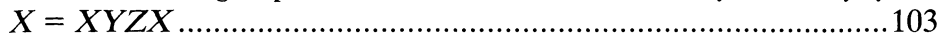

E. Grosswald, Rational valued series of exponentials and divisor

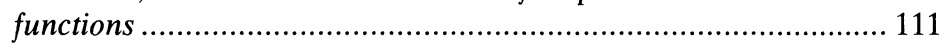

D. Handelman, Strongly semiprime rings ...................................... 115

J. N. Henry and D. C. Taylor, The $\bar{\beta}$ topology for $w^{*}$-algebras ............. 123

M. J. Hodel, Enumeration of weighted p-line arrays .......................... 141

S. K. Jain and S. Singh, Rings with quasiprojective left ideals .............. 169

S. Jeyaratnam, The diophantine equation $Y(Y+m)(Y+2 m) \times$

$$
(Y+3 m)=2 X(X+m)(X+2 m)(X+3 m) \ldots \ldots \ldots \ldots \ldots \ldots \ldots . . .183
$$

$\mathrm{R}$. Kane, On loop spaces without $p$ torsion .........................................189

Alvin J. Kay, Nonlinear integral equations and product integrals ..........203

A. S. Kechris, Countable ordinals and the analytic hierarchy, I ...........223

Ka-Sing Lau, A representation theorem for isometries of $C(X, E) \ldots \ldots . .229$

I. Madsen, On the action of the Dyer-Lashof algebra in $H_{*}(G)$..........235

R. C. Metzler, Positive linear functions, integration, and Choquet's theorem ........................................................................................ 277

A. Nobile, Some properties of the Nash blowing-up ............................297

G. E. Petersen and G. V. Welland, Plessner's theorem for Riesz conjugates 


\section{Pacific Journal of Mathematics}

\section{Vol. 60, No. $1 \quad$ September, 1975}

Donald Earl Bennett, Strongly unicoherent continua ................ 1

Walter Russell Bloom, Sets of p-spectral synthesis ................ 7

Richard Thomas Bumby and David Earl Dobbs, Amitsur cohomology of

quadratic extensions: formulas and number-theoretic examples .......

W. Wistar (William) Comfort, Compactness-like properties for generalized

weak topological sums ...................................

Dennis Robert Dunninger and John Stewart Locker, Monotone operators

and nonlinear biharmonic boundary value problems ..............

Theodore Erickson, Wallace Smith Martindale, III and J. Marshall Osborn,

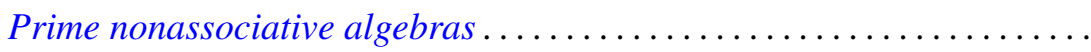

Pál Fischer, On the inequality $\sum_{i=0}^{n}\left[f\left(p_{i}\right) / f\left(q_{i}\right)\right] p_{i} \geq i \ldots \ldots \ldots \ldots \ldots$

Geoffrey Fox and Pedro Morales, Compact subsets of a Tychonoff set.......

Robert William Gilmer, Jr. and Joseph F. Hoffmann, A characterization of

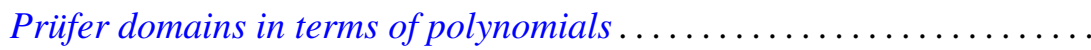

Leslie C. Glaser, On tame Cantor sets in spheres having the same projection

in each direction . ...................................

Zensiro Goseki, On semigroups in which $x=x y x=x z x$ if and only if

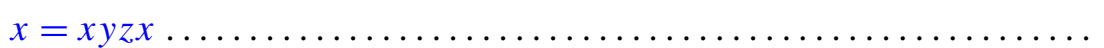

Emil Grosswald, Rational valued series of exponentials and divisor

functions.

David E. Handelman, Strongly semiprime rings

Jackson Neal Henry and Donald Curtis Taylor, The $\bar{\beta}$ topology for

$W^{*}$-algebras

Margaret Jones Hodel, Enumeration of weighted p-line arrays ...

Surender Kumar Jain and Surjeet Singh, Rings with quasi-projective left

ideals.

S. Jeyaratnam, The Diophantine equation

$$
Y(Y+m)(Y+2 m)(Y+3 m)=2 X(X+m)(X+2 m)(X+3 m) \ldots
$$

Richard Michael Kane, On loop spaces without $p$ torsion

Alvin John Kay, Nonlinear integral equations and product integrals ...

Alexander S. Kechris, Countable ordinals and the analytical hierarchy.

$I$.

Ka-Sing Lau, A representation theorem for isometries of $C(X, E)$

Ib Henning Madsen, On the action of the Dyer-Lashof algebra in $H_{*}(G)$

Richard C. Metzler, Positive linear functions, integration, and Choquet's

theorem.

Augusto Nobile, Some properties of the Nash blowing-up

Gerald E. Peterson and Grant Welland, Plessner's theorem for Riesz. 